\title{
WT1/PSMA/hTERT-encoding Plasmid DNA INO-5401
}

National Cancer Institute

\section{Source}

National Cancer Institute. WT 1/PSMA/hTERT-encoding Plasmid DNA INO-5401. NCI

Thesaurus. Code C148480.

A preparation composed of three separate DNA plasmids encoding the tumorassociated antigens (TAAs) Wilms tumor gene-1 (WT1), prostate-specific membrane antigen (PSMA) and human telomerase reverse transcriptase (hTERT), with potential immunostimulating and antineoplastic activites. Upon intramuscular delivery and electroporation of the WT1/PSMA/hTERT-encoding plasmid DNA INO-5401, the genes are translated into their respective proteins inside the cell. The expressed proteins activate the immune system and induce a cytotoxic T-lymphocyte (CT L)-mediated response against cells expressing the WT1, PSMA and hTERT antigens, causing tumor cell lysis. hTERT, WT 1 and PSMA are upregulated in many cancer cell types. 